\title{
Group Size and Direct Fitness in Social Queues
}

\author{
Gavin Shreeves $^{*}$ and Jeremy Field ${ }^{\dagger}$
}

Department of Biology, University College London, Wolfson House, London NW1 2HE, United Kingdom

Submitted November 27, 2000; Accepted July 15, 2001

ABSTRACT: We explore the effects of group size on the direct reproductive success of subordinate helpers in eusocial animals where only a single, dominant individual reproduces at one time. Helpers can reproduce directly if they inherit dominance, but when dominance is age based, an individual born into a larger group has a longer wait to inherit. We show that this disincentive to help can potentially be offset by increased productivity, increased life span, and insurance-based benefits for helpers if they survive to inherit dominance in larger groups. We analyze a field experiment in which group size was manipulated in the hover wasp Liostenogaster flavolineata. Productivity increased linearly with group size, larger groups were less likely to fail, and dominants in larger groups may have lived longer. Combined with the probability of inheriting dominance, these effects led overall to a negative correlation between group size and expected direct fitness, mainly because group size decreased during our study period, so that helpers could not expect to inherit as large a group as they started queuing in. Our analysis suggests that the relationship between group size and productivity plays a central role in determining the fitness consequences of helping.

Keywords: direct fitness, group size, queuing, eusociality, wasps, Stenogastrinae.

A key feature of some social groups is that they exhibit a reproductive division of labor in which some adults help to rear the offspring of others. We follow Gadagkar (1994) in referring to all such groups as "eusocial" to emphasize the similarities between disparate taxa, including insects and "cooperatively breeding" vertebrates (Emlen 1991). Work on both these taxa has played a key role in testing the predictions of kin selection theory (Hamilton 1963). Of particular interest are facultatively eusocial species, including most or all eusocial vertebrates, in which some

* E-mail: g-shreeves@yahoo.co.uk.

${ }^{\dagger}$ Corresponding author; e-mail: jeremy.field@ucl.ac.uk.

Am. Nat. 2002. Vol. 159, pp. 81-95. (c) 2002 by The University of Chicago. 0003-0147/2002/15901-0005\$15.00. All rights reserved. adult offspring disperse to attempt independent reproduction, while others remain in their natal groups as helpers.

In explaining the occurrence of helping behavior, studies of insects have often emphasized the degree of relatedness between helpers and the brood they rear and the indirect fitness benefits that result (e.g., Hamilton 1964; Trivers and Hare 1976; Seger 1983). However, studies of vertebrates have often suggested a role for direct reproductive success because an individual that is currently a nonbreeding subordinate may eventually inherit the dominant, reproductive position. This can provide a major incentive to stay, even when relatedness is very low (Reyer 1990; Stacey and Koenig 1990; Dunn et al. 1995; Queller et al. 2000). Increased direct benefits may be obtained by actively helping rather than simply waiting to inherit. This is because an active helper can boost group size so that she will have more helpers herself if she does inherit ("group augmentation"; Kokko et al. 2001). Recent twoplayer models have emphasized the potential importance of these direct benefits and suggest that animals will accept a subordinate role if their chance of inheriting the dominant position is high enough, even if the current dominant is unrelated and completely monopolizes reproduction (Kokko and Johnstone 1999; Ragsdale 1999).

In nature, animal societies are rarely limited to two individuals, and group size should have strong effects on an individual's decision of whether to stay in or disperse from a group (Higashi and Yamamura 1993). Because reproductive status is often contingent on social rank, the chance for any particular helper to attain a dominant, reproductive position will usually decrease as group size increases (e.g., Field et al 1999). However, larger groups may offer increased rewards for an individual that succeeds in becoming dominant. These large-group advantages can be divided into two main categories. First, a dominant in a larger group may have increased reproductive output, either because she has more helpers or through an increased life span because her helpers take over the risky foraging activities (Reeve 1991). Second, larger groups may have insurance-based advantages. These have been considered as indirect benefits to helpers and derive from the short life expectancy of adult caregivers in relation to the 
period of offspring dependency: offspring will often die without continuous adult care (Queller 1989, 1994; Gadagkar 1990; Reeve 1991; Field et. al. 2000). However, insurance-based advantages may also provide direct benefits to helpers that attain dominance; a larger group increases the chance that at least one adult will survive to tend the dominant's offspring through to independence.

In this article, we will examine the effects of group size on helpers' direct fitness when dominance is decided according to a gerontocracy, or age-based queue, in which the only breeder is the oldest member of the group. We note, however, that most of our conclusions can be generalized to other kinds of queues (see "Discussion"). Age is a convention for deciding dominance in diverse facultatively eusocial taxa, including many vertebrates, polistine and stenogastrine wasps, and halictine, allodapine, and xylocopine bees (e.g., in Hymenoptera, Strassmann and Meyer 1983; Samuel 1987; Hughes and Strassmann 1988; Yanega 1989; Kukuk and May 1991; Schwarz and Woods 1994; Hogendoorn and Velthuis 1995; Field et al. 1999; and in vertebrates, Wiley and Rabenold 1984; Stacey and Koenig 1990; Emlen 1991; Creel and Waser 1994; Dunn et al. 1995; Piper et al. 1995). Age-based queuing results in a strong, negative correlation between group size and a newborn helper's chance of inheritance. By joining the end of a longer queue, a helper faces a longer wait until she becomes dominant and is therefore more likely to die before she can reproduce directly (Field et al. 1999). However, she may also inherit large-group advantages if her group tends to remain large while she is queuing, countering the disadvantage of her longer wait in the queue. Direct fitness advantages are affected both by the probability of inheriting a resource and by the resource's value (Ragsdale 1999). Age-based queuing systems provide an especially clear illustration of how variation in group size can lead to a trade-off between these fitness components.

Our aim in this article is to examine whether helpers can expect to inherit large-group advantages and, if so, whether these advantages can compensate for their reduced chance of becoming dominant as group size increases. By examining the effects of group size on helpers' expectation of direct reproductive success and, therefore, their propensity to help, we are considering the circumstances when groups could form solely as societies of "hopeful reproductives" (West-Eberhard 1978; see also Walters et al. 1992). This is particularly relevant to facultatively eusocial vertebrates and Hymenoptera because they typically live in small groups. Helpers in such groups have a relatively good chance of inheriting dominance, and their position in the queue has a major impact on their expectation of future direct reproductive success (Field et al. 1999).

We will first present some simple, deterministic models that explore potential effects of group size on direct fitness in an age-based queuing system. Second, we will use Monte Carlo simulations to check the robustness of these deterministic models in the face of dynamic changes in group size. To provide an example of how to parameterize our models, we use data from a field experiment in which group size was manipulated in the facultatively eusocial hover wasp Liostenogaster flavolineata (Field et al. 2000). The experiment also allows us to test directly whether group size causally affects the survival rates of individual dominants and helpers and the failure rates of entire groups.

Liostenogaster flavolineata lives in small groups consisting of one behaviorally dominant female and zero to 10 subordinate helpers, with a mean group size of three or four females. Helpers defend the nest and forage to feed the brood (Samuel 1987). Although there are no morphological castes and subordinates are known to be capable of reproduction (Field and Foster 1999), the dominant female lays most or all of the eggs (Sumner et al., in press).

Each newly emerged female has two major options (Samuel 1987; Field et al. 1999). One is to leave and attempt to found a new nest on her own. The second is to remain in her natal group as a helper, in which case she joins the age-based queue for dominance and will eventually inherit the dominant position if she survives for long enough (Samuel 1987; Field et al. 1999; G. Shreeves, S. Sumner, M. Casiraghi, and J. Field, unpublished data). There is no clear colony cycle: brood-rearing occurs throughout the year, and groups can potentially persist indefinitely. Further details are given by Samuel (1987), Strassmann et al. (1994), and Field et al. (1998, 1999, 2000).

\section{Simple Models of Direct Reproductive Success}

\section{The Importance of Group Size}

We list all symbols and their definitions in table 1 . Consider a group of individuals in an age-based queue for dominance. Only the oldest individual in the group can reproduce at any one time, but any subordinate can potentially reproduce following the deaths of all individuals older than herself. Let $n$ be the total group size when a new female emerges. As the youngest female, she will rank the lowest in the queue, so that $n$ also represents her position in the queue. The probability that this individual will outlive all the individuals ahead of her is given by

$$
p_{n}=\frac{\mu_{n}}{\sum_{i=1}^{n} \mu_{i}}
$$


Table 1: Description of symbols used in models 1-3

\begin{tabular}{ll}
\hline Symbol & \multicolumn{1}{c}{ Description } \\
\hline$n$ & Group size and rank for a newborn helper \\
$g$ & Group size a helper expects to inherit if she becomes dominant \\
$p_{n}$ & Probability that a helper ranked $n$ becomes dominant \\
$B_{n}$ & Birth rate of new helpers in a group of size $n$ \\
$\beta$ & Proportionality coefficient between birth rate and group size, such that $B_{n}=\beta n$ \\
$F_{n, t}$ & Failure rate of a group of size $n$ over time $t$ \\
$f_{t}$ & Death rate of a single individual over time $t$, so that $F_{n, t}=f_{t}^{\mathrm{n}}$ if all individuals have the same death rate \\
$u_{d}$ & Expected life span of a dominant \\
$u_{f}$ & Expected life span of a helper \\
$\alpha$ & Relative gain in life span for a dominant compared to a helper, such that $u_{d}=\alpha u_{f}$ \\
$W_{1}$ & Expected direct fitness of a female nesting independently \\
$W_{n}$ & Expected direct fitness of a helper which starts at rank $n$
\end{tabular}

where $\mu_{i}$ is the expected life span of the $i$ th individual and the dominant has rank 1 (Syski 1979; Kokko and Sutherland 1998; Field et al. 1999). When all wasps have the same life span, the probability that the $n$th individual in a group becomes dominant is

$$
p_{n}=\frac{1}{n}
$$

Note that $p_{n}$ is independent of overall survivorship, whereas Kokko and Johnstone (1999) found that the two were positively correlated. This is because Kokko and Johnstone's (1999) model is based on discrete breeding seasons: a subordinate must survive until the next breeding season as well as outlive the dominant in order to reach the front of the queue.

If all dominants were to achieve the same direct reproductive output $(B)$ regardless of group size, a queuing female's expected direct reproductive success $\left(W_{n}\right)$ would decline with group size as

$$
W_{n}=\frac{B}{n}
$$

Since $W_{n}$ is maximized when $n=1$, females should prefer to leave and nest independently in the absence of indirect fitness benefits. We will consider three ways in which this decline in direct fitness might be overcome: increased productivity for dominants in larger groups, insurance benefits provided by larger groups, and increased life spans for dominants in larger groups.

\section{Model 1: Increased Productivity by Larger Groups}

A female that starts queuing in a large group may still be in a large group if she eventually becomes dominant. Helper death rates seem to be independent of group size in
Liostenogaster flavolineata (Field et al. 1999), so that if the rate of production of new helpers was proportional to group size (see below), group size could potentially remain stable. For simplicity, we will therefore assume that a dominant's reproductive output is proportional to her group size. If a helper that inherits dominance finds herself in a group of size $g$, then

$$
B_{g}=\beta g,
$$

where $\beta$ is the productivity added per wasp. When all wasps have the same expected life span, a helper starting at position $n$ in the queue has probability $1 / n$ of becoming dominant. If she does so, she will then be in a group of size $g$, where $g=n=1$ if she leaves to nest independently. Her expected direct reproductive success is then

$$
W_{n}=\frac{\beta g}{n} \text {. }
$$

Given that the reproductive success of nesting independently is $W_{1}=x \beta$, where $x$ represents any costs associated with dispersal and nest establishment $(0 \leq x \leq 1)$, this gives a simple condition for remaining in the queue of

$$
g \geq x . n
$$

The group size that a female expects to inherit should therefore have a strong influence on her propensity to stay. In the simplest case, where dispersal costs are negligible $(x=1)$, a female should be indifferent to queue length if she expects group size to remain stable $(g=n)$, should prefer to leave rather than queue if her group size is declining, and should be increasingly likely to queue if her group is growing. A nonlinear increase in productivity with group size will of course alter the precise result. For example, if productivity increases at a decelerating rate, there 
could be a more stringent condition for remaining in the queue. Conversely, the condition would be less stringent if nest establishment costs fall more heavily on individuals that leave.

\section{Model 2: Insurance Benefits in Larger Groups}

Immature offspring require continuous adult care in order to survive. A female that starts a new nest alone must therefore survive for at least the period of offspring dependency or she will obtain no reproductive success (Gadagkar 1990; Queller 1994; Field et al. 2000). However, lone foundresses in facultatively eusocial wasps seldom live this long, with failure rates being over $50 \%$ in L. flavolineata and many species of Polistes (Reeve 1991; Queller 1996; Field et al. 1998). Group living provides a number of potential insurance-based advantages that can ameliorate this demographic constraint. These have usually been considered in terms of indirect fitness benefits to helpers (e.g., Gadagkar 1990; Reeve 1991; Queller 1994; Field et al. 2000), but here we consider how a period of offspring dependency can affect a helper's expectation of direct fitness if she inherits the dominant egg-laying position. Specifically, the remaining group members could potentially save a dominant's brood even if she dies before those brood reach maturity.

Suppose that all wasps have the same expected life span and that dominants produce offspring at a rate independent of group size. However, now assume that a solitary foundress or a new dominant must wait for a time $t$ before any of her brood become adult. If all adults in the group die before this time, she will obtain no reproductive success, but if at least one survives we will assume her brood is saved. If the risk of each individual wasp dying before the larval development time $t$ is completed is $f_{t}\left(0<f_{t}<\right.$ 1 ), the risk of an entire group of $g$ wasps failing due to the death of all its members is

$$
F_{g, t}=f_{t}^{g}
$$

which decreases with increasing $g$ so that larger groups have a survival advantage. The direct reproductive success of a wasp that queues (and expects to inherit a group of size $g$ ) compared to a solitary nester is then (assuming negligible dispersal costs)

$$
\frac{1-f_{t}^{g}}{n .\left(1-f_{t}\right)} \text {. }
$$

Queuing is favored only when

$$
\left(1-f_{t}^{g}\right) \geq n \cdot\left(1-f_{t}\right)
$$

In a stable or declining group $(g \leq n)$, queuing can never be favored through this survival advantage alone. But if we add a linear increase in productivity with group size, as in model 1 , the two advantages combine. The condition for remaining in a queue is then

$$
\frac{g}{n} \geq \frac{1-f_{t}}{1-f_{t}^{g}} .
$$

In this case, remaining in a queue can be favored over independent nesting even if group size declines between the start of queuing and inheritance $(g<n$; fig. 1). The inherited group size above which waiting in the queue should be preferred decreases as the adult mortality rate $f_{t}$ increases (fig. 1).

\section{Model 3: Increased Life Span for Dominants in Larger Groups}

Adults in multifemale groups of most eusocial insects do not simultaneously expose themselves to what is probably their riskiest activity: foraging. When subordinate helpers are present, the dominant individual usually remains on the nest and relies on her subordinates to forage. As a

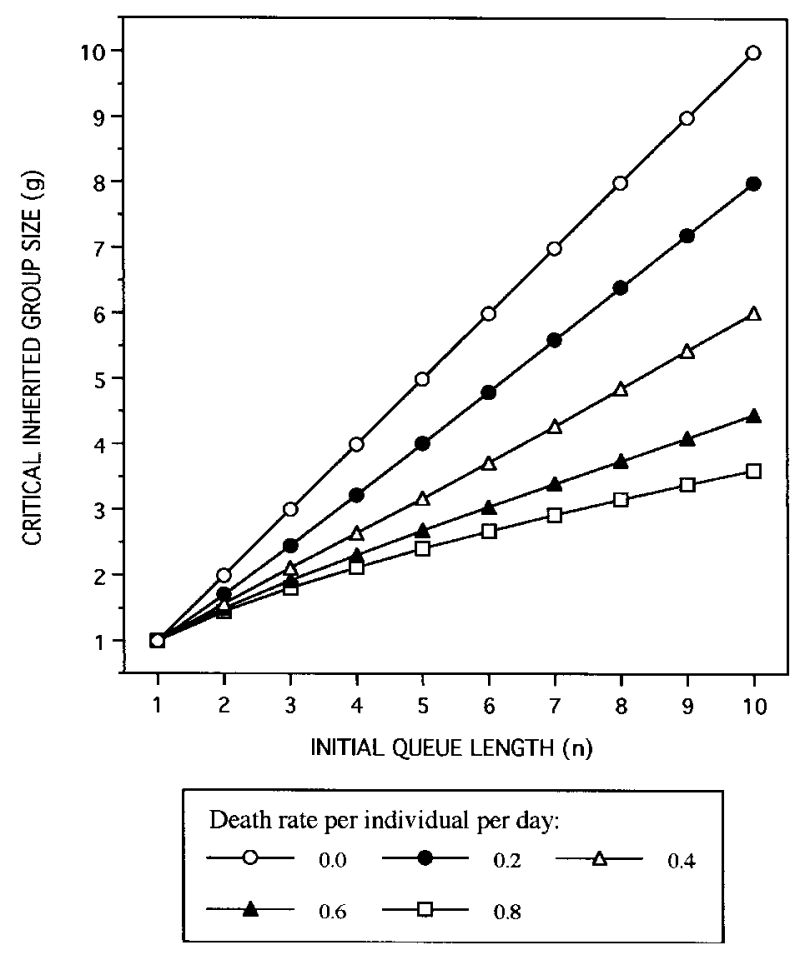

Figure 1: Critical group size $(g)$ that a female must expect to inherit if she is to join the end of a queue of length $n$ under model 2 (see text). The five lines represent different adult mortality rates. 
A.

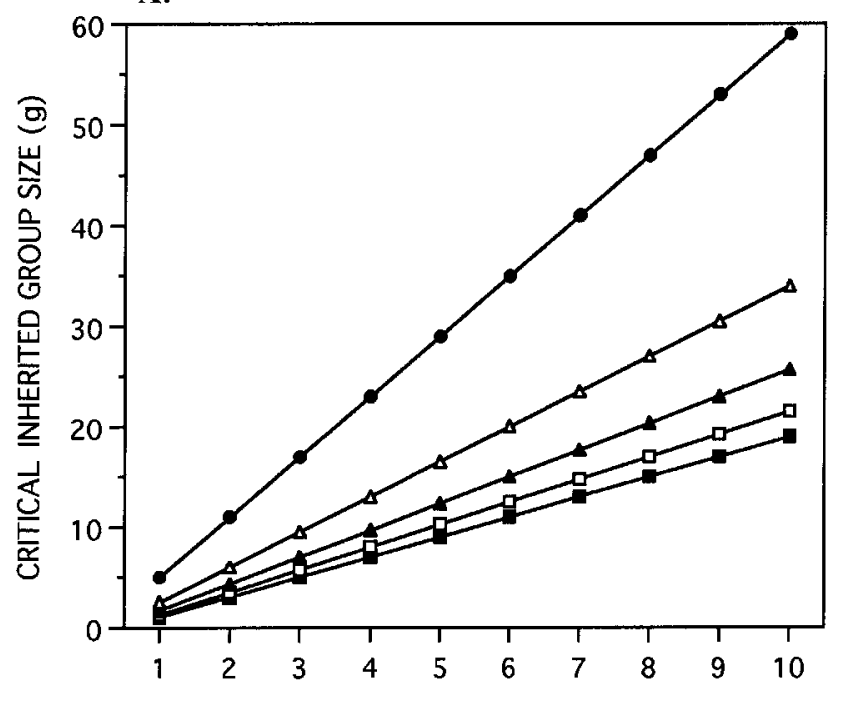

B.

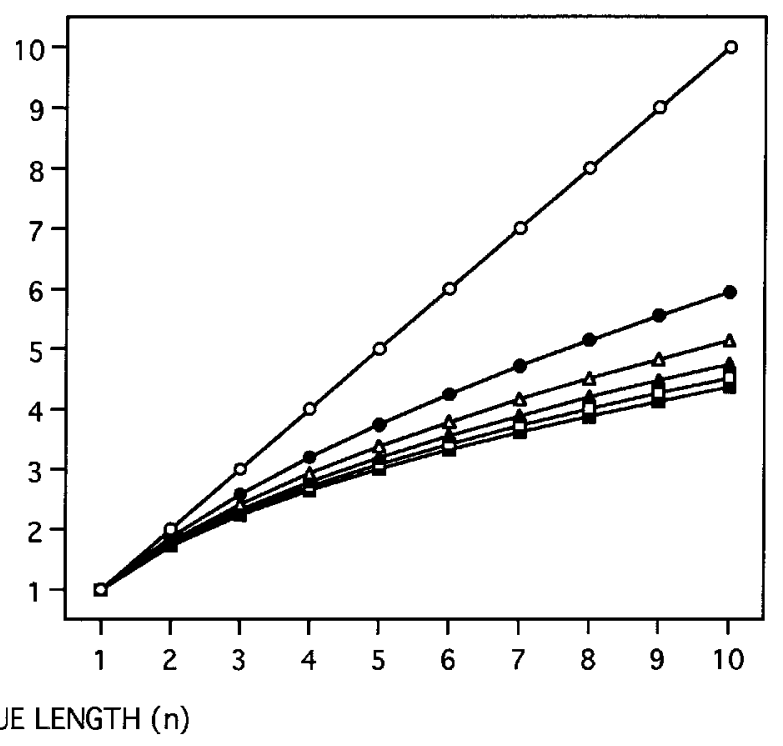

Increase in dominant's lifespan $(\alpha)$ :

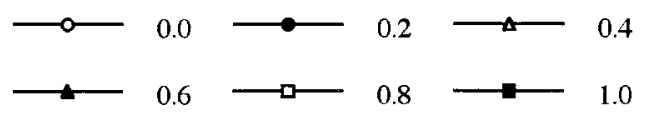

Figure 2: Critical inherited group size ( $g$ ) for joining a queue of length $n$ when a dominant's life span increases with group size (model 3; see text). $A$, Dominants in larger groups have increased life span but no additional productivity advantage. $B$, As in $A$, but dominants in larger groups now have an additional advantage because the rate of brood production is proportional to $g$.

result, her mortality is considerably reduced, compared to that of her helpers (Reeve 1991). When dominants live longer than their subordinates, the probability that the $n$th wasp in a queue of $n$ becomes dominant is, for $n>1$,

$$
p_{n}=\frac{u_{f}}{u_{d}+(n-1) \cdot u_{f}}
$$

where $u_{f}$ is the mean life span of each subordinate and $u_{d}$ is the dominant's mean life span. Suppose that solitary nesters have the same life span as a foraging helper, $u_{d}=u_{f}$, while, for each additional wasp in the group, a dominant gains extra life time of $\alpha . u_{f}$, then

$$
u_{d}=u_{f}+\alpha \cdot u_{f}(n-1)=u_{f}[\alpha(n-1)+1] .
$$

By substituting (12) into (11), the probability that the $n$th wasp in the queue will survive to become dominant can be written as

$$
p_{n}=\frac{1}{\alpha(n-1)+n}
$$

If dominants produce offspring at a constant rate $B$, independent of group size, then (ignoring insurance-based mechanisms) a queuing wasp that expects to inherit a group of size $g$ can expect direct reproductive success of

$$
\begin{aligned}
W_{n} & =B \cdot p_{n} \cdot u_{d} \\
& =B \cdot u_{f} \frac{\alpha(g-1)+1}{\alpha(n-1)+n} .
\end{aligned}
$$

This compares to a solitary female's reproductive success, assuming negligible dispersal costs, of

$$
W_{1}=B \cdot u_{f}
$$

Then, remaining in the queue should be preferred if

$$
g \geq \frac{n(\alpha+1)-1}{\alpha} .
$$

For $\alpha \geq 0$, this is true only if $g$ is greater than $n$ : for a wasp to stay, she must expect to inherit a larger group than she was born into (fig. $2 A$ ). The effect of a dominant's 
life span increasing with group size is to increase the length of time that subordinates must wait to inherit, causing a decrease in their expected direct reproductive success. The greater life span that a subordinate might get if she becomes dominant does not completely compensate for this.

However, an increase in productivity with group size can act synergistically to counteract this disadvantage. Combining a linear increase in brood production with group size, as in model 1, with a linear increase in dominants' life span with group size gives a condition for a female to remain in a queue of

$$
\begin{gathered}
\text { ק.g. } u_{f} \frac{\alpha(g-1)+1}{\alpha(n-1)+n} \geq \beta . u_{f}, \\
g(\alpha g-\alpha+1) \geq(\alpha n-\alpha+n) .
\end{gathered}
$$

This condition can be met under a variety of assumptions about $g$ (see fig. 2B). For example, if we assume stable group size $(g=n)$, joining a queue will always be preferred. Combining this final model with a survival advantage for larger groups (model 2) is complicated by the different life spans of dominants and subordinates and is most easily achieved using Monte Carlo simulations.

\section{Model 4: Monte Carlo Simulation}

The models above suggest that both initial group size $(n)$ and the group size that a helper expects to inherit $(g)$ should be of critical importance to her decision whether to queue. However, $g$ will clearly depend on how group size affects the balance between the production of new group members and the death rate of existing members. We have used Monte Carlo simulation models to investigate the relationship between $g$ and $n$ and to provide a test of whether the simple models' conclusions are valid in a more realistic situation where births and deaths can occur while helpers are queuing.

In this section, we describe these simulation models. We allow wasp survival rates, birth rates, and brood development time to vary as functions of group size. The simulations then allow us to estimate group failure rates, the probability that a helper becomes dominant, the mean group size if she does so $(g)$, and her expected direct reproductive success given that she starts as the $n$th helper in a group of size $n$. We provide the simulation results in the "Results" section, after a description of the methods we used to obtain empirical estimates for survival rates and birth rates in the wasp L. flavolineata.

Each replicate of our Monte Carlo simulation consists of following a group of simulated individuals over a number of discrete time steps, with each time step taken to be a single day. Total group size at the start of step $t+1$ is given by

$$
N_{t+1}=N_{t}+H_{t+1}-D_{t+1}
$$

where $H_{t+1}$ is a the number of new helpers that hatch during this time step and $D_{t+1}$ is the number of deaths. Both $H_{t+1}$ and $D_{t+1}$ are random variables, generated using algorithms given by Press et al. (1992).

For a newly established, single-female nest we assume that no new helpers can hatch until a period of development time has elapsed. Samuel (1987) estimated brood development time for L. flavolineata as $100 \mathrm{~d}$. We have assumed that after this time, new helpers hatch according to a Poisson process in which the rate parameter is proportional to group size at the end of the previous step:

$$
E\left[H_{t+1}\right]=\beta \cdot N_{t} .
$$

In contrast, we consider multifemale groups to be long established, so that they produce replacement helpers at this rate without first completing the period of development time. In order to simulate the death of dependent offspring in the absence of adult caregivers, we assume that no new helpers will hatch if all wasps in the simulated group have died before the end of a time step.

Our previous data suggest that wasps' life spans follow an exponential distribution (Field et al. 1999). In a discrete time simulation, this translates into a binomial distribution for the number of deaths per time step, in which the rate parameter depends on wasps' dominance status. The expected number of deaths in time step $t+1$ is then

$$
E\left[D_{t+1}\right]=1-S_{d}+\left(N_{t}-1\right)\left(1-S_{s}\right)
$$

where $S_{d}$ is the survival rate of the dominant per time step and $S_{s}$ is the survival rate of a subordinate. Using our empirical data, we have assumed $S_{s}$ to be constant with respect to group size, while $S_{d}$ depends deterministically on group size at the end of the previous time step, $N_{t}$. We use a logistic transformation for these parameters to ensure that survival rates remain between 0 and 1 .

To estimate the probability of a wasp becoming dominant, we follow a number of simulated individuals that start at rank $N$ in a group of size $N$. These will eventually either die or become dominant. If they become dominant, we record their reproductive success until death. We consider a wasp to be newly dominant if she is still alive at the end of a time step but all older individuals have died. A new dominant must then wait for the period of development time before any hatched offspring contribute to her direct fitness and is given zero direct fitness if all simulated group members die before this time. If a dominant 
dies with younger wasps still present, the new dominant could potentially replace all of the brood with her own or, at the opposite extreme, preserve all of her predecessor's brood. If a dominant in our simulations dies with younger wasps still present, we allow her one further time step of offspring production after her death. This probably underestimates the value of insurance provided by her surviving nestmates (Field et al. 2000).

\section{Methods for Estimating Parameter Values}

In this section, we describe methods used to obtain empirical parameter estimates for our models in the hover wasp Liostenogaster flavolineata. We use a removal experiment (Field et al. 2000) to estimate the relationships between group size and productivity and between group size and the survival rates of dominants, helpers, and entire groups.

Liostenogaster flavolineata is a convenient species to study because nests often occur in dense aggregations. From June to September 1998, we observed a population comprising four nesting aggregations (sites) in drainage culverts along $4 \mathrm{~km}$ of the road between Raub and Bukit Fraser in Selangor State, peninsular Malaysia (Field et al. 2000). These aggregations initially contained a total of 112 occupied nests.

During June 17-July 9, 1998, we individually marked almost all female nest residents using unique color combinations of enamel paints. Dominants rarely leave their nests (Field and Foster 1999) and were identified as the females most often present on nests during regular censuses conducted in the foraging period. Because most helpers return to their nests for the night, additional nest censuses carried out after dusk or just before dawn allowed us to estimate group size accurately.

\section{Removal Experiment}

In the models discussed above, the probability that a helper inherits dominance depends on group size and the survival rates of the females ahead of her in the queue. Once a helper has inherited dominance, insurance-based mechanisms may increase her reproductive success in larger groups. To test whether group size does have a causal effect on individual and group survival, we experimentally manipulated group size by removing one or two helpers from a random sample of nests (Field et al. 2000). We blocked nests within sites as closely as possible according to the total number of brood they contained (using intervals of 10 brood) and the number of marked residents alive just before the experiment. We then randomly assigned half of the nests in each category at each site to the removal treatment and the remainder to the control treatment. We removed two helpers from removal treatment nests with three or more residents and one helper from nests with two residents. Twenty nests had only a single resident at the start of the experiment and so could not be assigned to the removal treatment, leaving 45 nests in the control group and 47 in the removal group.

We removed helpers from nests in the removal treatment before dawn on July 9. We held a Ziploc bag under each nest, causing the wasps to drop into the bag. We retained the selected helpers and released their nestmates after dawn. Residents on the 45 control nests were captured in the same way, but all were then released. A few helpers that were absent on July 9 were removed over the next 2 d. We continued to census nests at night after performing the manipulation every $4 \mathrm{~d}$ until September 9, to record effects of the treatment on survival rates. Other aspects of this experiment are presented by Field et al. (2000).

Total failure of an L. flavolineata nest occurs if all residents disappear and the nest becomes vacant. Failure can also be assumed if the original occupants are replaced by wasp(s) from another nest because the brood are then generally eaten and replaced (Field et al. 1998, 2000). For the purposes of this article, we count wasp disappearances as entirely reflecting mortality, although some wasps undoubtedly emigrate (Field et al. 1999). For assessing nest failure rates, however, this is a reasonable simplification because wasps that emigrate no longer contribute to the maintenance of the nest or brood.

\section{Group Productivity}

To obtain estimates of group productivity, we mapped the cells in all nests and recorded the contents of each cell. We also estimated the size of brood in each cell, using three ordinal categories: pupae, medium- to large-sized larvae, and eggs plus tiny larvae combined. We updated the brood maps every 4-9 d until September 14 to record changes in brood number and brood development. Pupae are readily identified because pupal cells are closed with a conspicuous mud cap. The emerging adult destroys this cap, so that we could easily estimate hatching rates. It is possible that some pupae recorded as hatched had actually failed, but we also recorded the number of unmarked females present on nests after the start of the experiment. These were probably mostly newly emerged, and this allowed us to control for possible pupal deaths.

\section{Statistical Analysis}

For statistical analyses, apart from analysis of individual survival, we used general linear modeling in the GLIM statistical package (e.g., Crawley 1993). In each analysis, we first fitted all terms of interest, then subtracted terms 
until further removals led to significant $(P<.05)$ increases in deviance. We report significance levels for terms when adding them last to this minimal adequate model. When analyzing the effects of group size, we initially included both preremoval group size and treatment (control or removal) in the model. When both terms were significant, we assume that group size has a causal effect on the dependent variable. When preremoval group size but not treatment was significant, the interpretation was that a variable correlated with group size, but not group size itself, had a causal effect.

To analyze survivorship of individual wasps, we used a version of the Cormack-Jolly-Seber (CJS) mark-releaserecapture model (Jolly 1963; Cormack 1964; Seber 1965) in which we assumed that survival rates and the resighting probabilities for marked wasps did not change over time. As in a previous study (Field et al. 1999), we found these assumptions to be entirely reasonable for nighttime censuses in this population. Significance of terms in CJS models can be assessed analogously to general linear modeling in GLIM by calculating the change in the likelihood of a model caused by removing a term. Approximate standard errors for CJS model parameters can be produced either by using particular algorithms for maximizing the likelihood (see, e.g., Press et al. 1992) or by bootstrapping. Further details of the calculation of the likelihood for CJS models and the algorithms we used for maximizing it are discussed in Field et al. (1999; see also Pollock et al. 1990 and Lebreton et al. 1992).

\section{Results}

\section{Survival of Individuals: Dominance Status}

Survival rates of dominants were higher than those of their subordinates, with the overall mean daily survival rate for dominants assessed after the removals being $99.2 \%$, compared to $98.3 \%$ for subordinates. This corresponds to expected life spans ( \pm 1 approximate SE) of $132 \mathrm{~d}(110-156$ $\mathrm{d}, n=112)$ and $58 \mathrm{~d}(43-76 \mathrm{~d}, n=211)$, respectively. Survivorship of lone females was not significantly different from that of subordinates on multiple-female nests $(G=0.07, \mathrm{df}=1, P=.97)$.

\section{Group Size}

The removal treatment had no effect on subordinates' subsequent survival rates $(G=0.20, P=.65)$, and their survival was also not significantly affected by preremoval group size $(G=-0.63, P=.43)$. Their survival was negatively correlated with the total number of brood on their nests immediately before the experimental removals
( $G=6.96, P=.008, \mathrm{df}=1)$, and there was a marginal difference in survival between sites $(G=5.64, P=.060$, $\mathrm{df}=2$ ).

The effect of group size on dominants' survival rates was less clear. Although preremoval group size was positively correlated with dominants' subsequent survival rates (fig. 3; $G=6.007, \mathrm{df}=1, P=.01$ ), the experimental treatment itself had no significant effect $(G=2.83$, $\mathrm{df}=1, P=.09)$, so that a causal effect could not be confirmed. There was, however, a trend for dominants to have higher death rates on the removal nests (49\% over the postremoval observation period) than the controls (31\%). Given that observations ended before the overall median of survival was reached, it seems possible that statistical power was not high enough to detect a significant difference.

\section{Group Survival}

Groups with larger preremoval group sizes were significantly less likely to fail ( $G=25.9, \mathrm{df}=1, P<.001$ ). More importantly, however, the removal treatment significantly increased nest failure rates $(G=6.09, \mathrm{df}=1, P=.014)$, implying a causal relationship (fig. 4). During the 2 mo after the experimental removals, 17 out of $47(36 \%)$ of the removal nests failed, compared with eight out of 45 $(18 \%)$ of the control nests. The highest failure rate $(55 \%)$

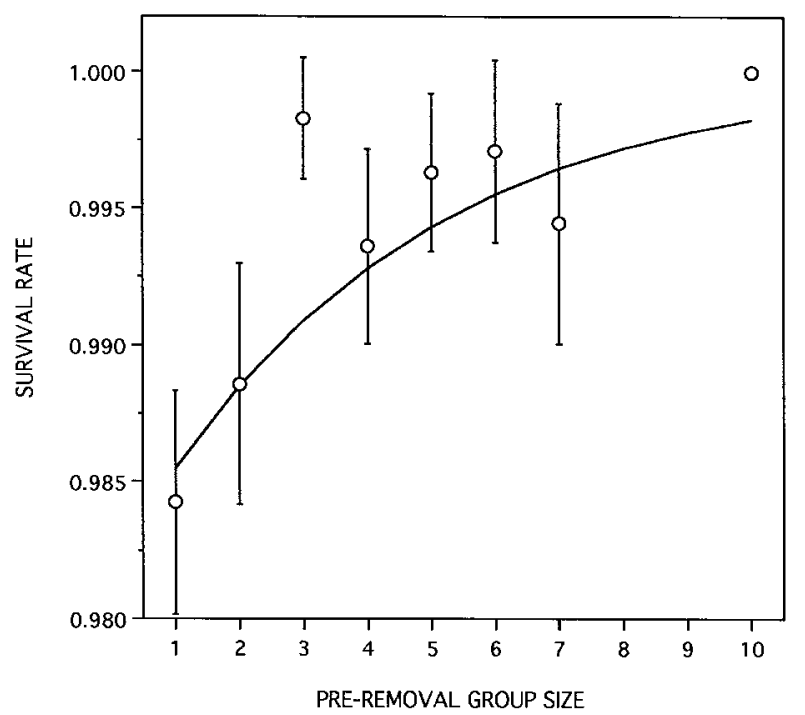

Figure 3: Survival of dominants in relation to group size. Dominants' survival estimated using a constant parameter Cormack-Jolly-Seber model for control and removal nests combined. Predicted values are inverse logits of $3.97+0.237 n$, where $n$ is group size immediately before the removal experiment. The error bars are \pm 1 SE for each group size, transformed from the logit scale. 


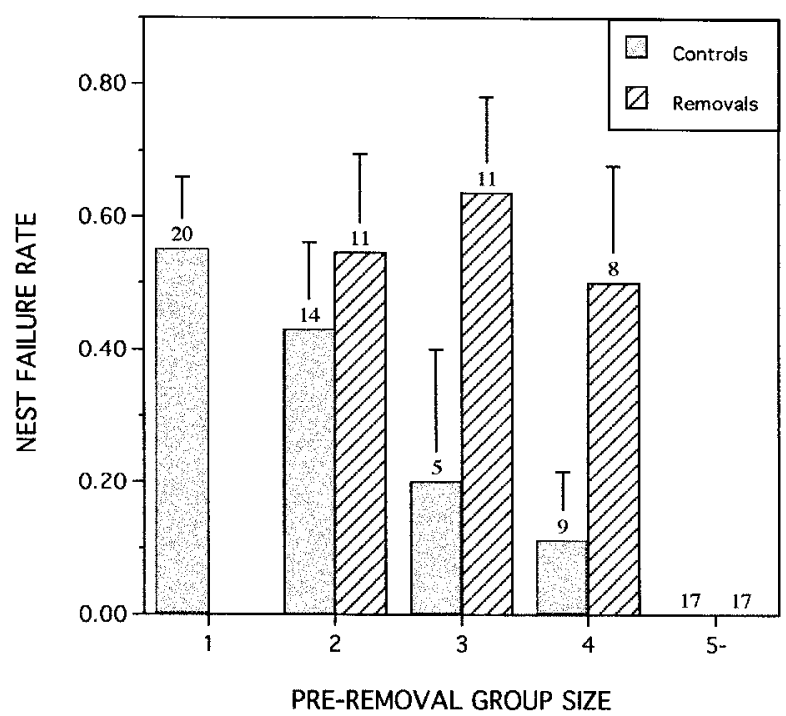

Figure 4: Failure rates of control, removal, and single-female nests in relation to preremoval group size. Bars show $1 \mathrm{SE}$; numbers below bars are sample sizes (number of nests).

occurred among the 20 nests with only a single occupant just before the removals. Group size declined gradually in the multiple-female nests that failed, and there was no evidence of mortality factors acting on whole groups at the nest. This suggests that nest failure resulted from independent deaths of all group members.

\section{Group Size and Productivity}

Productivity appeared to increase linearly with group size. The total number of brood of all stages increased linearly with group size just before the removals (regression is brood $=5.36$ [group size], $P<.0001, r^{2}=0.62$; see fig. 1 in Field et al. 2000). Estimated pupal hatching rates also increased linearly with group size, showing little sign of reaching a plateau at larger group sizes: adding a quadratic term or a nonzero intercept did not significantly improve the fit of the model. Approximately 0.7 additional pupae hatched per additional wasp over the $63 \mathrm{~d}$ from the start of our removal experiment until the end of observations (fig. 5).

There was no evidence that treatment affected the pupal failure rate. In total, we recorded 189 potentially hatched pupae and marked 141 new females, and treatment did not affect the proportion of new females to the number of potential emergences ( $G=0.098, \mathrm{df}=1, P=.75)$. This proportion also did not differ between sites $(G=$ 1.68 , $\mathrm{df}=2, P=.43$ ). The actual rate of recruitment of new helpers is likely to be lower than the pupal hatching rate because some of the emerging offspring will be males and because not all emerging females will remain as helpers (Field et al. 1999).

Group size declined over the course of our observations, suggesting that queuing wasps could not expect to inherit as large a group as they were born into. Excluding singlefemale nests, both preremoval group size and treatment significantly affected group size by the end of observations. Groups that were initially larger tended to remain larger $(F=10.1, \mathrm{df}=1,85, P=.002)$, while groups from which wasps had been removed remained smaller than control nests $(F=35.4, \mathrm{df}=1,85, P<.001)$. A quadratic term was significant for preremoval group size, indicating that larger groups declined to a relatively greater extent than small groups $(F=4.50, \mathrm{df}=1,85, P=.037)$. There were also differences between sites $(F=34.10, \mathrm{df}=$ $3,85, P<.0001)$, largely attributable to one site that did not decline as much as the others.

\section{Monte Carlo Simulation of Reproductive Success}

Probability of Becoming Dominant. Our data suggest mean survival rates for dominants of $132 \mathrm{~d}$, compared with 58 $\mathrm{d}$ for subordinates and lone females, with the dominant's survival rates also increasing with group size (fig. 3). Using the formula given in equation (11), the probability of a wasp ranked $n$ in a group becoming dominant should decline with $n$, as shown in figure 6. Monte Carlo simu-

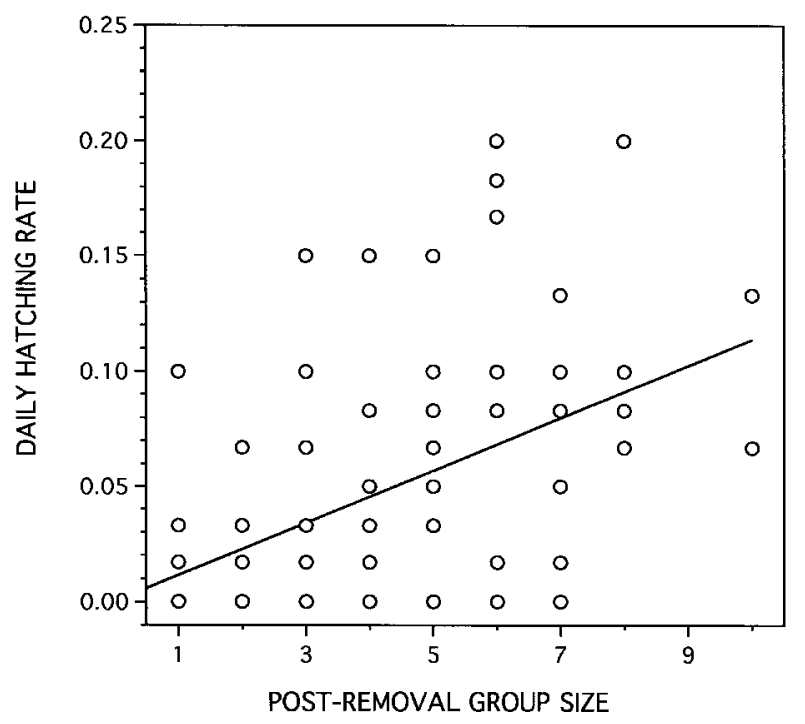

Figure 5: The pupal hatching rate over $63 \mathrm{~d}$ from the removal experiment on July 9, 1996, until the end of observations on September 15, 1996, for removal, control, and single-female nests combined. The fitted line is a linear regression with daily hatching rate $=0.011 n$, where $n$ is postremoval group size; $r^{2}=0.296$. 


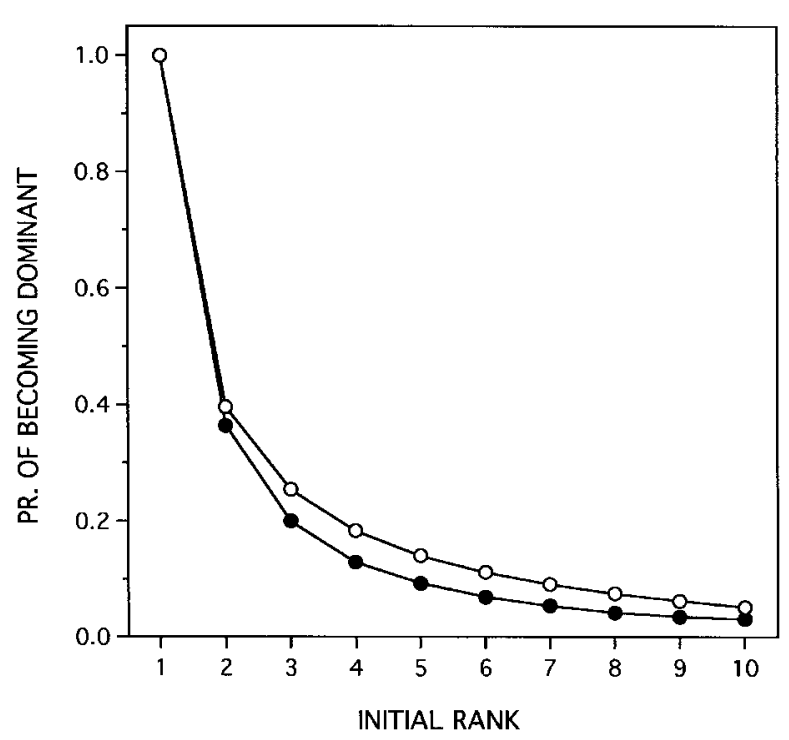

Figure 6: Estimated probability that a helper initially ranked $n$ in a queue becomes dominant. Open circles show data using the static formula from model 3, $p_{n}=u_{f} /\left[u_{d}+(n-1) \cdot u_{f}\right]$, where $u_{f}$ is the mean life span of a helper $(58 \mathrm{~d})$ and $u_{d}$ is the mean life span of a dominant, given by the fitted line $u_{d}=1+\exp (3.97+0.237 n)$. Filled circles show data from Monte Carlo simulation with survival rates per time step being $\exp (-1 / 58)=98.3 \%$ for helpers and $\exp \left[\left(3.97+0.237 n_{t}\right)\right] /[1+$ $\left.\exp \left(3.97+0.237 n_{t}\right)\right]$ for dominants, where $n_{t}$ is the group size at the start of time step $t$. The hatching rate assumed for new helpers is $0.011 n_{t}$, from the regression in figure 7 . Ten thousand replicates were run for each initial group size.

lations, which also allow for changes in group size due to births and deaths during the life of a dominant, produced very similar results (fig. 6). Running the simulation with a maximum time step of $63 \mathrm{~d}$ (our period of observations) suggested that it was giving reasonable results: with the distribution of group sizes immediately after the helper removals, the simulation suggested that we should expect to see 10.7 subordinates successfully inheriting dominant positions, compared with an observed total of 11 .

Rewards for Dominants. Our data show positive correlations between group size and dominants' survival (fig. 3) and group size and pupal hatching rates (fig. 5). In a static model, a dominant's total reproductive output should therefore increase as a quadratic function of group size. However, group size declined at three of our four sites over the course of our observations. Simulations show that a queuing wasp could expect to inherit a considerably smaller group than she was born into (fig. 7).

The decline in group size during our observations implies that a queuing female cannot necessarily expect to inherit a large enough group to compensate for her chance of death while waiting. Counteracting this effect, however, is increased group survival for larger groups (fig. 4). Monte Carlo simulations, which assumed that nest failure was caused entirely by independent deaths of group members, gave a close fit to the increase in nest survival with group size observed in control nests (fig. 8). For these simulations, we used Samuel's (1987) estimate of development time as $100 \mathrm{~d}$. We assumed three possible rates of production of replacement helpers, ranging from zero (which would be the case for a new nest before the completion of the first offspring development) to the full pupal hatching rate, estimated from the regression on group size shown in figure 5 .

Overall, combining the probability of becoming dominant (fig. 6) with rewards for dominants (figs. 9, 10), the simulations suggest that a wasp's expected direct fitness should decline with queue length in our population. If all offspring are female and become helpers, the second female in a group of two has the same expected direct reproductive success as a solitary nester, but lower-ranked wasps should expect greater direct fitness if they left to establish their own nests (fig. 9). Apart from group size, two main factors determine the relative advantage of staying rather than leaving to nest independently. First, reducing brood development time makes leaving to nest independently increasingly more profitable (fig. 9). Second, increasing the rate at which pupae hatch per day increases helpers' expected direct fitness (fig. 10). As pupal

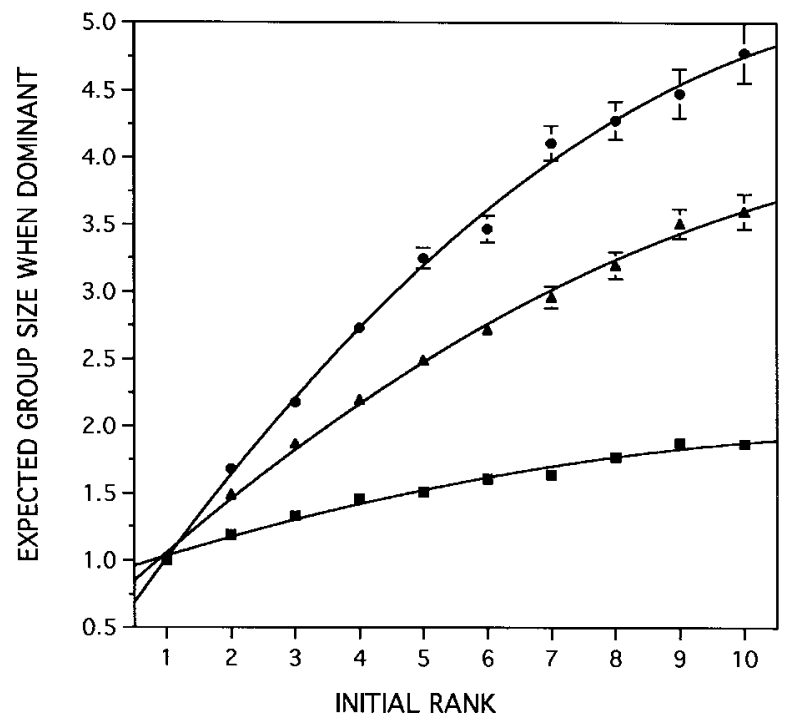

Figure 7: Simulated group size $(g)$ that a helper initially ranked $n$ in a queue will inherit if she becomes dominant, for three different hatching rates of new helpers: $0.015 n_{t}$ (circles), $0.011 n_{t}$ (triangles), and each queue length (squares). 


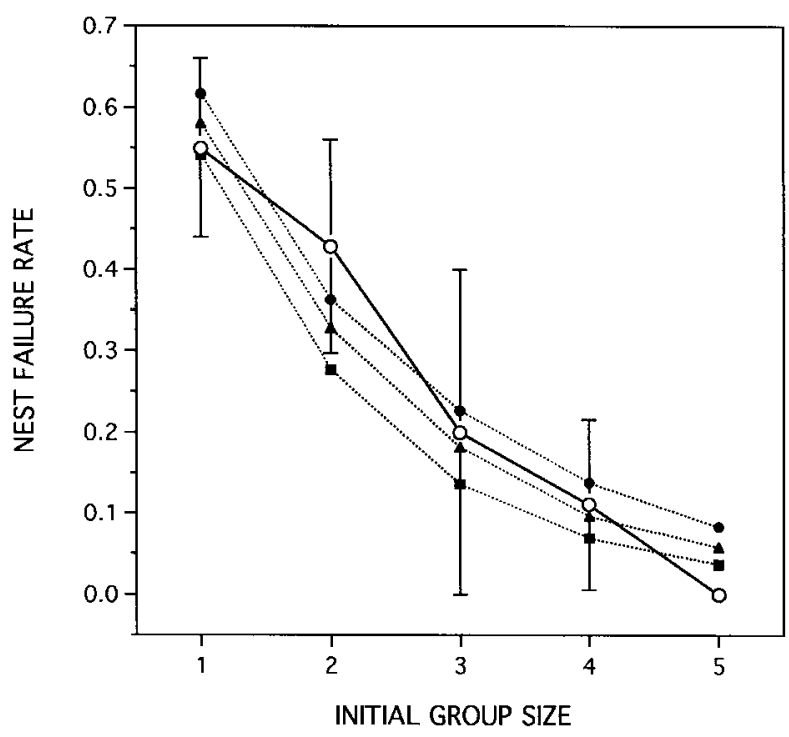

Figure 8: Actual nest survival \pm 1 SE (open circle) and simulated nest survival for differing initial group sizes over $63 \mathrm{~d}$ of observations. Simulated survival rates come from 5,000 Monte Carlo replicates per group size, using our estimates for wasp survival rates, with production rates of new helpers as follows: no births (closed circle); total number of pupae hatching taken from the regression in figure 5 (square); half of the preceding rate, to allow for losses due to potential helpers leaving and male production (triangle).

hatching rates per group member increase, helpers in larger groups gain an increasing direct fitness advantage as they expect to inherit a larger, more productive group if they become dominant.

\section{Discussion}

In facultatively eusocial animals, a single dominant may monopolize offspring production at any one time. The probability that a particular subordinate inherits the dominant position will be negatively correlated with the number of individuals that the subordinate is competing with. We have focused on the common case of a new group member in an age-based queue: her probability of attaining dominance will be approximately inversely proportional to group size (Field et al. 1999). It is worth noting, however, that group size can have the same effect when other conventions decide dominance. For example, in a group composed of individuals from a random size distribution, the chance that any particular individual is the largest is again inversely proportional to group size (G. Shreeves, unpublished data). We would therefore often expect increasing group size to reduce a given individual's chance of obtaining direct reproductive success.

We have examined three direct fitness advantages of inheriting a larger group that could potentially compensate for a reduced probability of inheriting: increased productivity in a larger group, a longer life span for dominants in larger groups, and insurance benefits provided by larger groups. Of these, we suggest that increased productivity by larger groups has the potential to maintain helping behavior at any queue length (model 1). By contrast, a longer life span for dominants in larger groups increases the wait to inherit and cannot by itself increase a helper's expected direct fitness (see also Kokko and Johnstone 1999). It can, however, act synergistically with productivity to promote group living (model 3). Insurance benefits increase at a diminishing rate with increasing group size and are therefore not alone sufficient to promote group living at larger group sizes.

Our models also suggest that helpers' direct fitness will depend critically on the population dynamics within their groups. In the simplest case, where productivity increases proportionally with group size and group size is stable, helpers can expect the same direct fitness despite starting from the ends of different-sized queues. However, helpers that expect group size to have declined by the time they

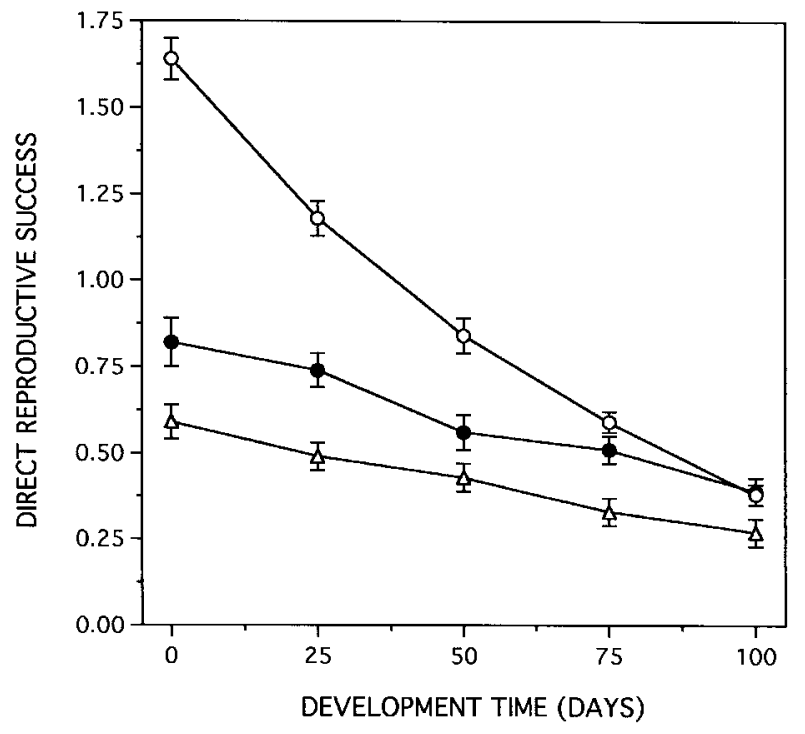

Figure 9: Effect of development time on expected direct reproductive success. Expected direct reproductive success for solitary females (open circle) and wasps starting at the ends of queues of length 2 (filled circle) or 3 (open triangle), with development time varying from 0-100 d (100 $\mathrm{d}$ is the observed value for Liostenogaster flavolineata). For solitary females, no replacement helpers are allowed to hatch before the end of the development time. Expected reproductive success takes into account the probability of the group surviving over this development time (fig. 8), expected group size when dominant (fig. 7), and the probability of becoming dominant (fig. 6). Data from 10,000 Monte Carlo replicates; wasp survival and hatching-rate parameters as for figure 6. 


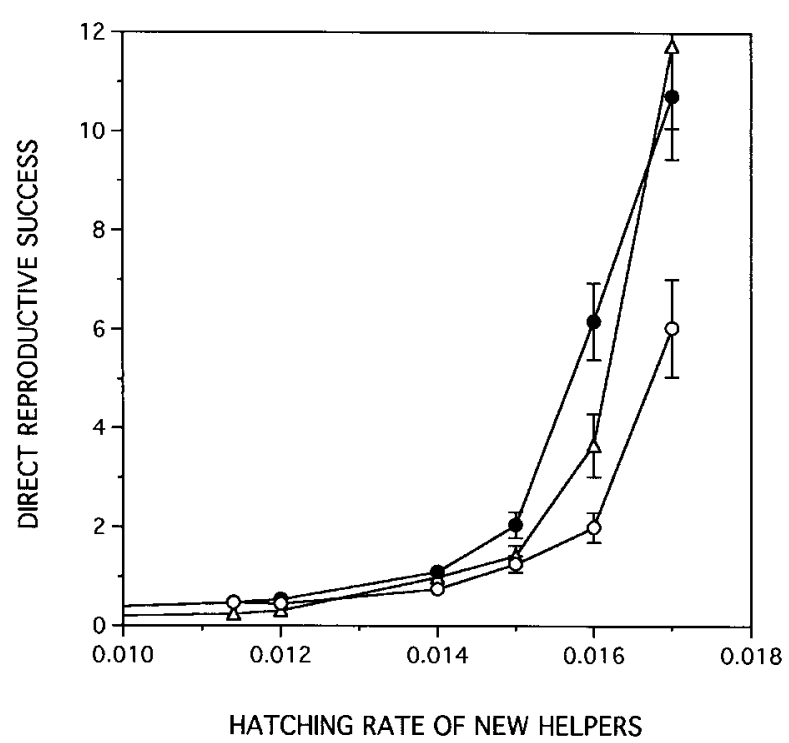

Figure 10: Effect of helper hatching rate on expected direct reproductive success. Hatching rates of new helpers taken as $0.005 n_{t}$ (open circle), $0.011 n_{t}$ (open triangle), and $0.015 n_{t}$ (filled circle) per day, where $n_{t}$ is group size at the start of time interval $t$. Our field data suggest a rate of at most $0.011 n_{t}$ per day. Expected reproductive success includes the probability of the group surviving over a development time of $100 \mathrm{~d}$ (fig. 8), expected group size when dominant (fig. 7), and the probability of becoming dominant (fig. 6). Data from 10,000 Monte Carlo replicates; wasp survival parameters as for figure 6 .

inherit dominance require larger benefits in order for queuing to remain worthwhile. This suggests a possible answer to a problem in the evolution of helping pointed out by Queller (1994): under many circumstances, an optimal strategy for a subordinate is not to increase her mortality rate by actively helping but, rather, to do as little as possible while waiting to inherit the group. If, however, a subordinate's future reproductive success depends partly on her own efforts as a helper, the advantage of a pure waiting strategy is reduced (Kokko et al. 2001).

\section{Direct Fitness in Eusocial Animals}

In any particular species, the precise relationship between group size and a helper's expectation of direct fitness will obviously depend on the form of the relationship between group size and productivity. In eusocial vertebrates, group productivity may increase with group size at an accelerating or decelerating rate (Johnstone et al. 1999). In eusocial insects, however, it is traditionally believed that the relationship is decelerating, that is, that per capita productivity declines with increasing group size (Michener 1964; Wenzel and Pickering 1991). In the framework we have developed, this means that although dominants in larger groups may still have greater productivity, a helper might not inherit a large enough group for direct fitness alone to compensate for waiting in a longer queue. However, Michener's (1964) paradox is by no means universal. We have found that total number of brood increases linearly with group size in two out of three populations of Liostenogaster flavolineata studied (Field et al. 1999; this study; J. Field, G. Shreeves, and S. Sumner, unpublished data). Estimated pupal hatching rates also increased linearly with group size in this study. Linear or accelerating relationships between per capita productivity and group size have also been found in other Hymenopterapolistine wasps (Shakarad and Gadagkar 1995; Jeanne and Nordheim 1996 [see also Karsai and Wenzel 1998]; M. Cant and J. Field, unpublished manuscript), allodapine bees (Schwarz 1988; Schwarz et al. 1998), and halictine bees (Packer 1993). Furthermore, because most studies have not taken failure during nest establishment into account, the relative productivity of smaller groups may have been overestimated.

Because insect caregivers probably have higher mortality rates than vertebrates, insurance-based advantages may apply particularly to insects (Queller 1996). As in L. flavolineata, there is little evidence that larger group size in facultatively eusocial Hymenoptera provides accelerating group survival benefits through increasingly effective nest defense (Reeve 1991; Queller 1996, but see Strassmann et al. 1988). Instead, the major cause of nest failure is usually the successive independent deaths of group members, combined with whole-nest predation acting randomly with respect to group size. Under these circumstances, each additional helper adds only a diminishing increment to nest survival (Nonacs 1991), and survival insurance is not sufficient by itself to maintain a queue of hopeful reproductives (model 2).

The effect of group size on the survivorship of dominants has been little investigated. In some vertebrates, however, there is correlative evidence that helpers do increase the survivorship of breeders (Emlen 1991).

\section{Direct Fitness in Liostenogaster flavolineata}

In a previous study of L. flavolineata, we found no correlation between group size and the rate at which newly emerged females disappeared from their natal nests (Field et al. 1999). This suggested that there must be advantages of being in larger groups that counteract the smaller chance of inheriting from the end of a longer queue. Our experimental data show that dominants in larger groups are more productive and live longer (fig. 3, 5). There is also a positive correlation between group size and group survival (fig. 4). These advantages would be sufficient to maintain a group of two females through expected direct 
fitness benefits alone (figs. 9, 10). However, the mean group size in our population was four females, and we must infer that additional advantages are often needed to maintain helping behavior in this population. Nestmates in L. flavolineata are usually related, and indirect fitness benefits of helping are undoubtedly important (Field et al. 1998, 2000).

A major factor that reduced the direct benefits obtained by helpers in larger groups was the decline in group sizes during our study period. As illustrated by our models, a helper that queues to inherit a declining group risks dying without obtaining any direct reproductive success and stands to receive a smaller benefit if she does eventually inherit. The reasons for the decline in our study population are not clear but may possibly be seasonal: although peninsular Malaysia does not have an extreme monsoonal climate, there are distinct dry and wet seasons (e.g., Samuel 1987). Our observations were made during the dry season, and we also observed a decline in group size over the dry season in a previous study (July-September 1996; Field et al. 1999) and a subsequent increase by the start of the wet season (November; G. Shreeves, personal observation). We speculate that if productivity per wasp declines predictably during the dry season, the benefits of leaving to reproduce immediately might outweigh those of waiting to inherit what will be a less productive group in the future. If more offspring leave, group size declines, further reducing the benefits of inheritance. This feedback loop could be reversed as the wet season approaches.

Our simulations of direct fitness in L. flavolineata are simplified in several respects. In particular, they assume that individuals have exponential survivorship and that changes in group size are immediately tracked by changes in the hatching rate of replacement helpers. The first of these assumptions seems to be valid, at least over the time span of our observations (see also Field et al. 1999). The second assumption is certainly not strictly correct: with a larval development time of $100 \mathrm{~d}$ (Samuel 1987), there is likely to be a lag between changes in group size and changes in hatching rates. However, our simulation results agreed closely with our observational data in predicting the number of helpers that inherited dominance during the course of our observations and in predicting nest failure rates (fig. 8). This suggests that the simulations have succeeded in modeling the essential features of this system.

\section{Conclusion}

The small group sizes and age-based queuing systems seen in facultatively eusocial vertebrates and Hymenoptera imply that direct reproduction via inheritance is often an important component of helper inclusive fitness. Among Hymenoptera, this may be particularly true of tropical species in which waiting times are less constrained by the seasons. In temperate wasps and bees, the short season may truncate the life spans of initially low-ranking helpers before all of their older nestmates have died.

Our models suggest a central role for the relationship between group size and productivity in determining the fitness consequences of helping. This relationship has a considerable impact both on group dynamics and on the rewards for inheriting dominance. Although group members may not always act as foragers, group size may still determine the quantity or quality of resources available to breeders, including helpers that eventually inherit breeding status. Our models suggest that a simple proportional increase in reproductive rate for dominants of larger groups could compensate for helpers' reduced chance of inheriting.

Assuming that helpers' survival does not increase with increasing group size, the survival rate of groups increases at a decelerating rate with increasing group size and therefore cannot act by itself to maintain levels of direct benefits for helpers in larger groups (model 2). Similarly, although dominants may have longer life spans in larger groups, this increases the wait to inherit and cannot by itself increase a helper's expected direct fitness (model 3). Both of these mechanisms can, however, act synergistically with productivity increases. Given stable group sizes and increasing productivity with group size, our models suggest that direct fitness benefits alone have the potential to provide a sufficient staying incentive for helpers at any group size.

\section{Acknowledgments}

We thank M. Casiraghi and S. Sumner for help with fieldwork and A. Bourke, M. Cant, I. Karsai, H. Kokko, J. Ragsdale, J. Strassmann, and an anonymous referee for commenting on the manuscript. This research was funded by a Natural Environment Research Council research grant to J.F.

\section{Literature Cited}

Cormack, R. M. 1964. Estimates of survival from the sighting of marked animals. Biometrika 51:429-438.

Crawley, M. J. 1993. GLIM for ecologists: methods in ecology. Blackwell Scientific, Oxford.

Creel, S. R., and P. M. Waser. 1994. Inclusive fitness and reproductive strategies in dwarf mongooses. Behavioral Ecology 5:339-348.

Dunn, P. O., A. Cockburn, and R. A. Mulder. 1995. Fairywren helpers often care for young to which they are unrelated. Proceedings of the Royal Society of London B, Biological Sciences 256:339-343.

Emlen, S. T. 1991. Evolution of cooperative breeding in 
birds and mammals. Pages 301-337 in J. R. Krebs and N. B. Davies, eds. Behavioural ecology: an evolutionary approach. 3d ed. Blackwell Scientific, Oxford.

Field, J., and W. Foster. 1999. Helping behaviour in facultatively eusocial hover wasps: an experimental test of the subfertility hypothesis. Animal Behaviour 57: 633-636.

Field, J., W. Foster, G. Shreeves, and S. Sumner. 1998. Ecological constraints on independent nesting in facultatively eusocial hover wasps. Proceedings of the Royal Society of London B, Biological Sciences 265:973-977.

Field, J., G. Shreeves, and S. Sumner. 1999. Group size, queuing and helping decisions in facultatively eusocial hover wasps. Behavioral Ecology and Sociobiology 45: 378-385.

Field, J., G. Shreeves, S. Sumner, and M. Casiraghi. 2000. Insurance-based advantage to helpers in a tropical hover wasp. Nature (London) 404:869-871.

Gadagkar, R. 1990. Evolution of eusociality: the advantage of assured fitness returns. Philosophical Transactions of the Royal Society of London B, Biological Sciences 329: $17-25$.

- 1994. Why the definition of eusociality is not helpful to understand its evolution and what should we do about it. Oikos 70:485-488.

Hamilton, W. D. 1963. The evolution of altruistic behaviour. American Naturalist 97:354-356.

. 1964. The genetical evolution of social behaviour. I, II. Journal of Theoretical Biology 7:1-52.

Higashi, M., and N. Yamamura. 1993. What determines animal group size? insider-outsider conflict and its resolution. American Naturalist 142:553-563.

Hogendoorn, K., and H. H. W. Velthuis. 1995. The role of young guards in Xylocopa pubescens. Insectes Sociaux 42:427-448.

Hughes, C., and J. E. Strassmann. 1988. Age is more important than size in determining dominance among workers in the primitively eusocial wasp, Polistes instabilis. Behaviour 107:1-14.

Jeanne, R. L., and E. V. Nordheim. 1996. Productivity in a social wasp: per capita output increases with swarm size. Behavioural Ecology 7:43-48.

Johnstone, R. A., R. Woodroffe, M. A. Cant, and J. Wright. 1999. Reproductive skew in multimember groups. American Naturalist 153:315-331.

Jolly, G. M. 1963. Estimates of population parameters from multiple recapture data with both death and dilutiondeterministic model. Biometrika 50:113-128.

Karsai, I., and J. W. Wenzel. 1998. Productivity, individuallevel and colony-level flexibility, and organization of work as consequences of colony size. Proceedings of the National Academy of Sciences of the USA 95: 8665-8669.
Kokko, H., and R. A. Johnstone. 1999. Social queuing in animal societies: a dynamic model of reproductive skew. Proceedings of the Royal Society of London B, Biological Sciences 266:571-578.

Kokko, H., and W. Sutherland. 1998. Optimal floating and queuing strategies: consequences for density dependence and habitat loss. American Naturalist 152: 354-366.

Kokko, H., R. A. Johnstone, and T. H. Clutton-Brock. 2001. The evolution of cooperative breeding through group augmentation. Proceedings of the Royal Society of London B, Biological Sciences 268:187-196.

Kukuk, P. F., and B. May. 1991. Colony dynamics in a primitively eusocial halictine bee Lasioglossum (Dialictus) zephyrum (Hymenoptera: Halictidae). Insectes Sociaux 38:171-189.

Lebreton, J. D., K. P. Burnham, J. Clobert, and D. R. Anderson. 1992. Modeling survival and testing biological hypotheses using marked animals: a unified approach with case studies. Ecological Monographs 62: 67-118.

Michener, C. D. 1964. Reproductive efficiency in relation to colony size in hymenopterous societies. Insectes Sociaux 4:317-342.

Nonacs, P. 1991. Alloparental care and eusocial evolution: the limits of Queller's head start advantage. Oikos 61: 122-125.

Packer, L. 1993. Multiple foundress associations in sweat bees. Pages 215-233 in L. Keller, ed. Queen number and sociality in insects. Oxford University Press, Oxford.

Piper, W. H., P. G. Parker, and K. N. Rabenold. 1995. Facultative dispersal by juvenile males in the cooperative stripe-backed wren. Behavioral Ecology 6:337-342.

Pollock, K. H., J. D. Nichols, C. Brownie, and J. E. Hines. 1990. Statistical inference for capture-recapture experiments. Wildlife Monographs 107.

Press, W. H., S. A. Teukolsky, W. T. Vetterling, and B. P. Flannery. 1992. Numerical recipes in C: the art of scientific computing. $2 \mathrm{~d}$ ed. Cambridge University Press, Cambridge.

Queller, D. C. 1989. The evolution of eusociality: reproductive head starts of workers. Proceedings of the National Academy of Sciences of the USA 86:3224-3226. . 1994. Extended parental care and the origin of eusociality. Proceedings of the Royal Society of London B, Biological Sciences 256:105-111.

. 1996. The origin and maintenance of eusociality: the advantage of extended parental care. Pages 218-234 in S. Turilazzi and M. J. West-Eberhard, eds. Natural history and evolution of paper-wasps. Oxford University Press, Oxford.

Queller, D. C., F. Zacchi, R. Cervo, S. Turillazzi, M. T. Henshaw, L. A. Santorelli, and J. E. Strassman. 2000. 
Unrelated helpers in a social insect. Nature (London) 405:784-787.

Ragsdale, J. E. 1999. Reproductive skew extended: the effect of resource inheritance on social organization. Evolutionary Ecology Research 1:859-874.

Reeve, H. K. 1991. Polistes. Pages 99-148 in K. G. Ross and R. W. Matthews, eds. The social biology of wasps. Cornell University Press, Ithaca, N.Y.

Reyer, H.-U. 1990. Pied kingfishers: ecological causes and reproductive consequences of cooperative breeding. Pages 527-558 in P. B. Stacey and W. D. Koenig, eds. Cooperative breeding in birds. Cambridge University Press, Cambridge.

Samuel, C. T. 1987. Factors affecting colony size in the stenogastrine wasp Liostenogaster flavolineata. Ph.D. diss. University of Malaya.

Schwarz, M. P. 1988. Local resource enhancement and sex ratios in a primitively eusocial bee. Nature (London) 331:346-348.

Schwarz, M. P., and R. E. Woods. 1994. Order of adult eclosion is a major determinant of reproductive dominance in the allodapine bee Exoneura bicolor. Animal Behaviour 47:373-378.

Schwarz, M. P., N. J. Bull, and K. Hogendoorn. 1998. Evolution of sociality in the allodapine bees: a review of sex allocation, ecology and evolution. Insectes Sociaux 45:349-368.

Seber, G. A. F. 1965. A note on the multiple recapture census. Biometrika 52:249-259.

Seger, J. 1983. Partial bivoltinism may cause alternating sex-ratio biases that favour eusociality. Nature (London) 301:59-62.

Shakarad, M., and R. Gadagkar. 1995. Colony founding in the primitively eusocial wasp, Ropalidia marginata (Hymenoptera: Vespidae). Ecological Entomology 20: 273-282.

Stacey, P. B., and W. D. Koenig. 1990. Splendid fairy wrens: demonstrating the importance of longevity. Pages 1-30 in P. B. Stacey and W. D. Koenig, eds. Cooperative breeding in birds. Cambridge University Press, Cambridge.
Strassmann, J. E., and D. C. Meyer. 1983. Gerontocracy in the social wasp, Polistes exclamans. Animal Behaviour 31:431-438.

Strassmann, J. E., D. C. Queller, and C. R. Hughes. 1988. Predation and the evolution of sociality in the paper wasp Polistes bellicosus. Ecology 69:1497-1505.

Strassmann, J. E., C. R. Hughes, S. Turillazzi, C. Solis, and D. C. Queller. 1994. Genetic relatedness and incipient eusociality in stenogastrine wasps. Animal Behaviour 48: 813-821.

Sumner, S., M. Casiraghi, W. Foster, and J. Field. In press. High reproductive skew in tropical hover wasps. Proceedings of the Royal Society of London B, Biological Sciences.

Syski, R. 1979. Random processes: a first look. Vol. 29. Statistics: textbooks and monographs. Dekker, New York.

Trivers, R. L., and H. Hare. 1976. Haplodiploidy and the evolution of the social insects. Science (Washington, D.C.) 191:249-263.

Walters, J. R., P. D. Doerr, and J. H. Carter, III. 1992. Delayed dispersal and reproduction as a life-history tactic in cooperative breeders: fitness calculations from redcockaded woodpeckers. American Naturalist 139: 623-643.

Wenzel, J. W., and J. Pickering. 1991. Cooperative foraging, productivity, and the central limit theorem. Proceedings of the National Academy of Sciences of the USA 88: 36-38.

West-Eberhard, M. J. 1978. Polygyny and the evolution of social behaviour in wasps. Journal of the Kansas Entomological Society 51:832-856.

Wiley, R. H., and K. N. Rabenold. 1984. The evolution of cooperative breeding by delayed reciprocity and queuing for favourable social positions. Evolution 38:609-621.

Yanega, D. 1989. Caste determination and differential diapause within the first brood of Halictus rubicundus in New York (Hymenoptera: Halictidae). Behavioral Ecology and Sociobiology 24:97-107.

Associate Editor: Joan E. Strassman 